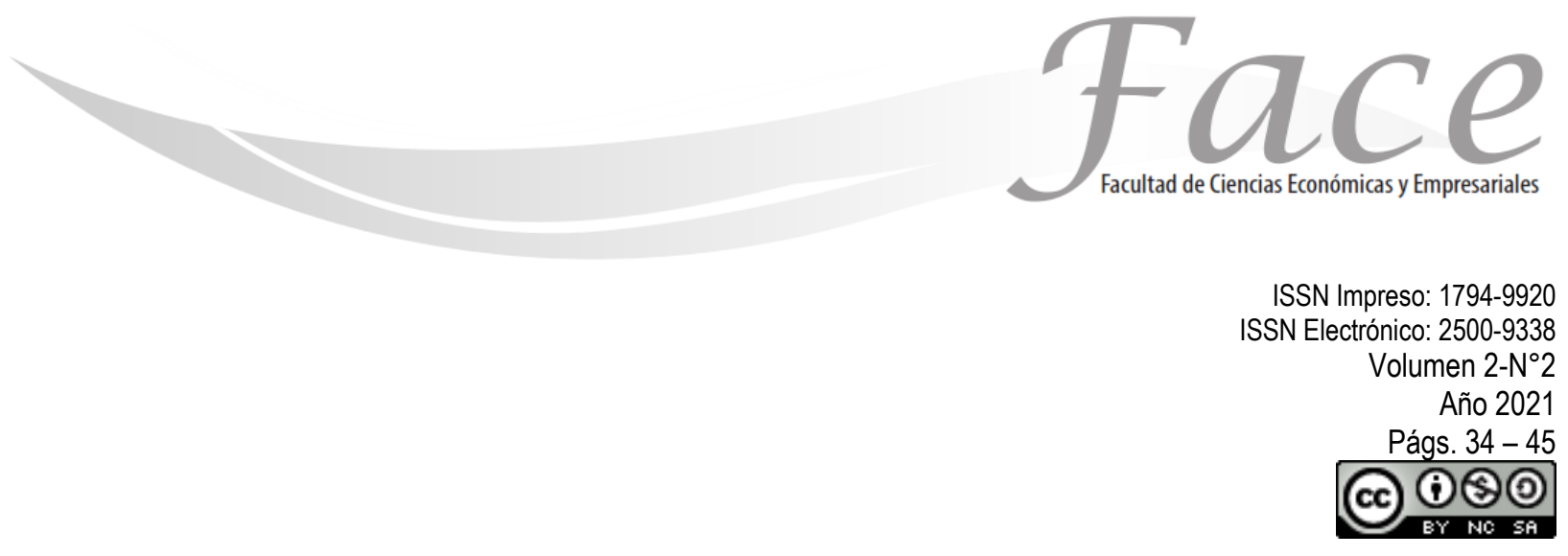

\title{
INFLUENCIA DE LAS VARIABLES DEMOGRAFICAS Y LA RESPONSABILIDAD SOCIAL EMPRESARIAL EN LA COMPRA DE LOS COLOMBIANOS
}

\author{
*Diana María López Celis \\ Enlace ORCID: http://orcid.org/0000-0002-7949-0625 \\ **Mónica Eugenia Peñalosa Otero \\ Enlace ORCID: http://orcid.org/0000-0002-2208-9224
}

Fecha de Recepción: Febrero 14

Fecha de Aprobación: Mayo 23

\section{Resumen:}

Este trabajo busca determinar la influencia que tienen o podrían llegar a tener las variables demográficas, sumado a los programas de Responsabilidad Social Empresarial (RSE), en la disposición de compra de los consumidores colombianos en productos que realicen dichas prácticas, evaluando las dimensiones de cuidado ambiental, cuidado social/ético y trato con los empleados; desde las variables demográficas el género, la edad, nivel de educación y ocupación. El tipo de investigación realizada fue concluyente con alcance cuantitativo, la medición se hizo con base en la batería de frases actitudinales correspondientes a la (RSE); el análisis se realizó a través de regresiones logísticas. El estudio arrojó un mayor aporte de las 3 dimensiones de la (RSE) con mayor participación de la variable ambiental, respecto a las variables social y ética, y un bajo porcentaje de aporte por parte de las variables demográficas; lo cual indica que es viable la construcción de un modelo de influencia con las dimensiones de los programas de RSE sobre la disposición a pagar más, pero no de la misma manera para la influencia de las variables demográficas.

Palabras clave: Compra, responsabilidad social, consumo, Colombia.

*Profesora Asociada de la Facultad de Ciencias Económicas y Administrativas, Universidad Jorge Tadeo Lozano, Bogotá, Colombia. Magister en Psicología del Consumidor, especialista en Psicología del Consumidor. Profesional en Psicología. Investigadora Jr. de Colciencias. Se ha venido desempeñando en los últimos años como Docente - Investigadora en la Universidad Jorge Tadeo Lozano, la Universidad Central y la Universidad Javeriana- Colombia. Correo electrónico: diana.lopez@utadeo.edu.co

**Profesora Asociada de la Facultad de Ciencias Económicas y Administrativas, Universidad Jorge Tadeo Lozano, Bogotá, Colombia. Magister en Mercadeo Agroindustrial. Profesional en Relaciones Internacionales. Investigadora Jr. de Colciencias. Se ha venido desempeñando en los últimos años como Docente Investigadora en la Universidad Jorge Tadeo Lozano, la Universidad de La Salle y la Universidad de Pamplona - Colombia. Correo electrónico: monica.penalosa@utadeo.edu.co 


\title{
SOCIAL MARKETING IN HEALTH IN TIMES OF COVID-19
}

\begin{abstract}
:
This paper seeks to determine the influence that demographic variables have or could have, added to the Corporate Social Responsibility (CSR) programs, on the willingness of Colombian consumers to purchase products that carry out said practices, evaluating the dimensions of care environmental, social / ethical care and dealing with employees; from the demographic variables gender, age, level of education and occupation. The type of research carried out was conclusive with a quantitative scope, the measurement was made based on the battery of attitudinal phrases corresponding to the (RSE); the analysis was carried out through logistic regressions.

The study showed a greater contribution of the 3 dimensions of the (CSR) with greater participation of the environmental variable, with respect to the social and ethical variables, and a low percentage of contribution by the demographic variables; which indicates that the construction of an influence model with the dimensions of CSR programs on the willingness to pay more is feasible, but not in the same way for the influence of demographic variables.
\end{abstract}

Keywords: Purchase, social responsibility, consumption, Colombia

\section{MARKETING SOCIAL EM SAÚDE NA ÉPOCA DA COVID-19}

\section{Resumo:}

Este trabalho busca determinar a influência que as variáveis demográficas têm ou poderiam ter, somadas aos programas de Responsabilidade Social Corporativa (RSC), sobre a disposição dos consumidores colombianos de adquirir produtos que realizem tais práticas, avaliando as dimensões do cuidado ambiental, social / cuidado ético e trato com os funcionários; a partir das variáveis demográficas sexo, idade, escolaridade e ocupação. 0 tipo de pesquisa realizada foi conclusivo com escopo quantitativo, a mensuração foi feita com base na bateria de frases atitudinais correspondentes ao (RSE); a análise foi realizada por meio de regressões logísticas.

O estudo mostrou uma maior contribuição das 3 dimensões da (RSC) com maior participação da variável ambiental, no que diz respeito às variáveis sociais e éticas, e um baixo percentual de contribuição pelas variáveis demográficas; o que indica que a construção de um modelo de influência com as dimensões dos programas de RSE sobre a disposição a pagar mais é viável, mas não da mesma forma para a influência das variáveis demográficas.

Palavras-chave: Compra, responsabilidade social, consumo, Colômbia. 


\section{INTRODUCCIÓN:}

La Responsabilidad Social (RSE) tiene cada día un papel más importante en la sociedad, dado que las prácticas de producción sostenibles son clave, tanto para el avance de las naciones como para el desarrollo de la economía mundial; donde se garantice el bienestar de la comunidad en general. La (RSE) nace desde una perspectiva corporativa como una necesidad para la sociedad, en donde busca que las acciones que la empresa lleve a cabo se encuentren armonizadas con su entorno; dado que ésta, ha sido una herramienta que por años ha prevalecido dentro de las empresas en diferentes sectores de la economía; su implementación se ha desarrollado tanto por decisión propia de los directivos y como parte de su ADN, a tal punto que ya existen empresas que integran estos programas como parte de su filosofía de negocio.

Dentro de ese nuevo paradigma, actualmente se pueden encontar las también llamadas Empresas $B$, las cuales operan bajo altos estándares sociales, ambientales, de transparencia y de acuerdo con sus estatutos, toman decisiones corporativas legalmente vinculantes considerando no sólo los intereses financieros de sus accionistas, sino también otros intereses y de largo plazo tales como la de los empleados, los proveedores y clientes, la comunidad a la que pertenecen y el medio ambiente. También pueden ser implementados estos programas motivados por la legislación de diferentes países, donde los programas de (RSE) son de carácter obligatorio con el fin de proteger a todos los actores del proceso.

A medida que el consumidor deja de pensar en forma individual y empieza a pensar de forma colectiva, surgen otras variables a considerar en el proceso de compra tal como es el caso de la dimensión de consumo responsable, la cual influye en el proceso de decisión y elección de las marcas. Según Havas Worldwide (2016), se encuentra que el $58 \%$ de los consumidores se interesan por el impacto social y ambiental de las marcas. De acuerdo con el Foro Económico Mundial (2017), se determinó las percepciones de los jóvenes acerca del mundo; se ratificó que los llamados "millennials" están preocupados especialmente, por los efectos que ha tenido el cambio climático con respecto al medio ambiente. Es así, como de los 31.000 encuestados el 48,8\% respondió que el cambio climático y la destrucción de la naturaleza es uno de los asuntos más graves a nivel global. A este problema le siguieron los grandes conflictos sociales con un porcentaje de $38,9 \%$, y en tercer lugar se ubicó la desigualdad y la discriminación con un porcentaje de 30,8\%. Lo anterior supone, una generación con mayor conciencia ambiental, lo que significa que están cambiando los hábitos de compra y como consumidores están siendo analíticos frente a sus acciones con el medio ambiente.

Teniendo en cuenta el impacto de las variables en referencia en la compra de productos, es pertinente analizar en el contexto colombiano, qué intención tienen los consumidores a pagar más por marcas socialmente responsables, teniendo en cuenta la influencia que puedan a llegar a tener las variables demográficas en esta actitud.

\section{MARCO TEÓRICO}

Disposición en los consumidores a pagar más por productos socialmente responsables

El impacto de la (RSE) en las marcas, no solamente ha sido objeto de estudio para la cultura occidental, también es tema de investigación para la cultura oriental, Tian, Wang \& Yang (2011), determinaron que "los consumidores con un mayor nivel de conocimiento y confianza de la (RSE) de las empresas, eran más propensos a pasar de una evaluación positiva de ellas a una intención de compra positiva". Para Arredondo, Maldonado \& de la Garza (2011), es mucho más frecuente encontrar que las mujeres apoyen las 
causas de (RSE), dado que a la hora de analizar sus percepciones y actitudes de compra en el marco de campañas de marketing con causa. Estos hallazgos soportan el impacto positivo del uso de los programas de (RSE). (Marquina Feldman \& Reficco, 2015); mientras que para Cai \& Aguilar (2013), existe una predisposición positiva a pagar precios extra por productos social y ambientalmente certificados, específicamente, estarían dispuestos a pagar un $12,2 \%$ más de su valor real por este tipo de productos; así como el $65 \%$ de los encuestados sienten responsabilidad para adquirir productos positivos para el medio ambiente y la sociedad.

Según Amezcua, Briceño, Rios \& Ayala (2018), el consumidor de hoy está dispuesto a pagar un sobreprecio aproximado del $22 \%$ por productos líderes en la categoría estudiada asociados a campañas con causa social, en comparación a un $10 \%$ en productos menos posicionados. Este efecto no está presente en campañas ambientales. De esta forma, se demuestra que las campañas sociales crean beneficios económicos para la empresa mientras que la sociedad se beneficia de la participación del consumidor en este tipo de campañas. Hoy las empresas están en la búsqueda de consolidar las preferencias de los consumidores hacia los productos fabricados por ellos, de tal manera que estos bienes y servicios se posesionen no solo en la mente sino en el corazón de los consumidores. Osorio, L. M. B., Andrade, J. M. M., \& Mina, L. G. (2015).

Específicamente en Colombia, al realizar la revisión teórica, solamente se encontraron tres (3) investigaciones respecto a la dinámica que tiene la (RSE) con respecto a la decisión de compra. El primero realizado en la ciudad de Bogotá titulado "Impacto de la responsabilidad social empresarial en el comportamiento de compra y disposición a pagar de consumidores bogotanos" (Percie y Reficco 2015), el segundo, "Disposición a pagar por atributos de responsabilidad social empresarial Un estudio aplicado a jóvenes de Medellín" (Solari, E. \& Pacheco Ortiz, D, 2015) y por último "Diseño y Responsabilidad Social Empresarial en la planeación estratégica de la marca", realizado por (Alzate y Orozco, 2015). Estos 3 estudios son coincidentes en sus resultados, los cuales demuestran que los consumidores de un lado consideran el atributo de responsabilidad social en su decisión de compra y de otro lado estiman que el manejo de la (RSE) dentro de la planeación estratégica ayuda en la construcción de marca.

De acuerdo con la última medición llevada a cabo en 2015 entre las principales empresas colombianas, se refleja que cuando se pregunta sobre el valor que aporta la (RSE) a la empresa, respecto a la reputación/valor de marca, el 22,3\%, considera que aporta a la construcción de marca, según la Asociación Nacional de Empresarios de Colombia. Para Castelló (2010), la (RSE) debe ser parte integral de las estrategias de branding corporativo. Según Villafañe (2009), "la (RSE) se ha convertido en parte importante de la comunicación y construcción de marca, puesto que, en la medida en que se gestionen adecuadamente los diferentes aspectos que hacen parte de la relación empresa-comunidad o marca-comunidad, es posible generar una imagen positiva en la mente de los consumidores", citados por (Alzate, J. E., \& Orozco-Toro, J. A., 2015). Para Licandro, Alvarado, Sansores \& Navarrete (2019), la interpretación que tienen los tomadores de decisiones en las empresas sobre el concepto de (RSE), es un factor crítico para su incorporación a la praxis empresarial; de esa interpretación depende el grado de transversalidad con el que se lo aplica y la forma como se lo gestiona.

\subsection{La segmentación demográfica y la disposición para pagar más por productos socialmente responsables.}

En la actualidad, existen estudios sobre el análisis de las variables demográficas y su incidencia en la actitud de los consumidores sobre el consumo socialmente responsable; sin embargo, es incipiente la información sobre la influencia de las variables demográficas y la disposición de los 
consumidores a pagar más por productos socialmente responsables. Como se observó en la revisión de la literatura, no se puede concluir si las variables demográficas realmente inciden en la actitud de los consumidores socialmente responsables, puesto que depende más del contexto donde interactúe el individuo. Teniendo en cuenta que existen diferentes variables que pueden afectar la medición del consumo socialmente responsable y las discrepancias que pueden existir, se hace difícil obtener a nivel global una estandarización para la segmentación del consumidor socialmente responsable en cuanto a variables sociodemográficas, de ahí la importancia de realizar estudios transculturales 0 multiculturales respecto al tema y la segmentación a nivel psicográfico.

Para Vicente y Mediano, (2002), este hecho es especialmente relevante si se tiene en cuenta que existen cada vez más indicios sobre la descripción tradicional del consumidor ecológico, como una persona joven, bien educada y de alto nivel de ingresos, se está desvaneciendo en favor de un consumidor de naturaleza y características cada vez más diversas. Según Zhilong Tian, Rui Wang, Wen Yang, (2011), la relación entre las variables demográficas y el consumo socialmente responsable no son lineales; mientras que para Pérez y Rodríguez (2015), las variables demográficas de género, edad y nivel educativo no permiten identificar diferencias en la forma en que los clientes construyen las imágenes de CSR. Se puede concluir que las variables demográficas, no son diferenciadoras en la segmentación de clientes para el diseño de mejores estrategias de (RSE) y la comunicación.

López \& Peñalosa (2018); señalan que, el consumo socialmente responsable se encuentra relacionado con las características personales y conductuales en un 69\%; mediado por las variables de consumo ético, conocimiento del medio ambiente y actitudes del medio ambiente. Respecto al aporte de las variables demográficas, no se encontró un aporte a la identificación 0 caracterización de este consumidor; debido a que, al incluir estas variables en el modelo estadístico determinado, muy por el contrario de potencializarlo, el nivel de ajuste del modelo con las variables incidentes presentó una disminución. El hallazgo anterior, no quiere decir que no se deben considerar las variables geo-demosociodemográficas, pero igualmente no son variables que determinen la conducta del consumidor socialmente responsable.

Por lo anterior, al no contar con un análisis concluyente acerca de las variables demográficas que inciden en el comportamiento de compra de los consumidores con un enfoque socialmente responsable, es necesario estudiar las variables relacionadas con el consumidor en sí, desde el aspecto psicosocial, teniendo en cuenta actitudes, motivaciones, estilos de vida, preocupación por el medio ambiente, valores, personalidad, entre otras. (Sánchez 2014).También hay que tener presente que en al actualidad, la productividad de los trabajadores, además del clima laboral en el que estén inmersos, está ligada a las compensaciones e incentivos que ofrece la organización, lo cual genera espacios de crecimiento en todos los aspectos y, a su vez, redunda en mejores resultados para la organización. Otalvaro, M. J. N. (2016).

Finalmente, de acuerdo la revisión de la literatura cobra gran relevancia que, en Colombia, se analice la intención de los consumidores a pagar más por marcas socialmente responsables y si las variables demográficas tienen alguna influencia en esta actitud.

\section{METODOLOGÍA}

Este trabajo busca dar respuesta a la siguiente pregunta: ¿Influyen las variables demográficas en la disposición de los consumidores colombianos a pagar más por marcas/ productos que realicen prácticas de responsabilidad social empresarial? Para tal fin, se estableció como objetivo, determinar la influencia de las variables 
demográficas en la disposición de los consumidores colombianos a pagar más por marcas/ productos que realicen prácticas de responsabilidad social empresarial.

\section{Hipótesis de trabajo}

H1: Existe influencia entre las variables demográficas y la disposición de los consumidores colombianos a pagar más por marcas / productos que realicen prácticas de responsabilidad social.

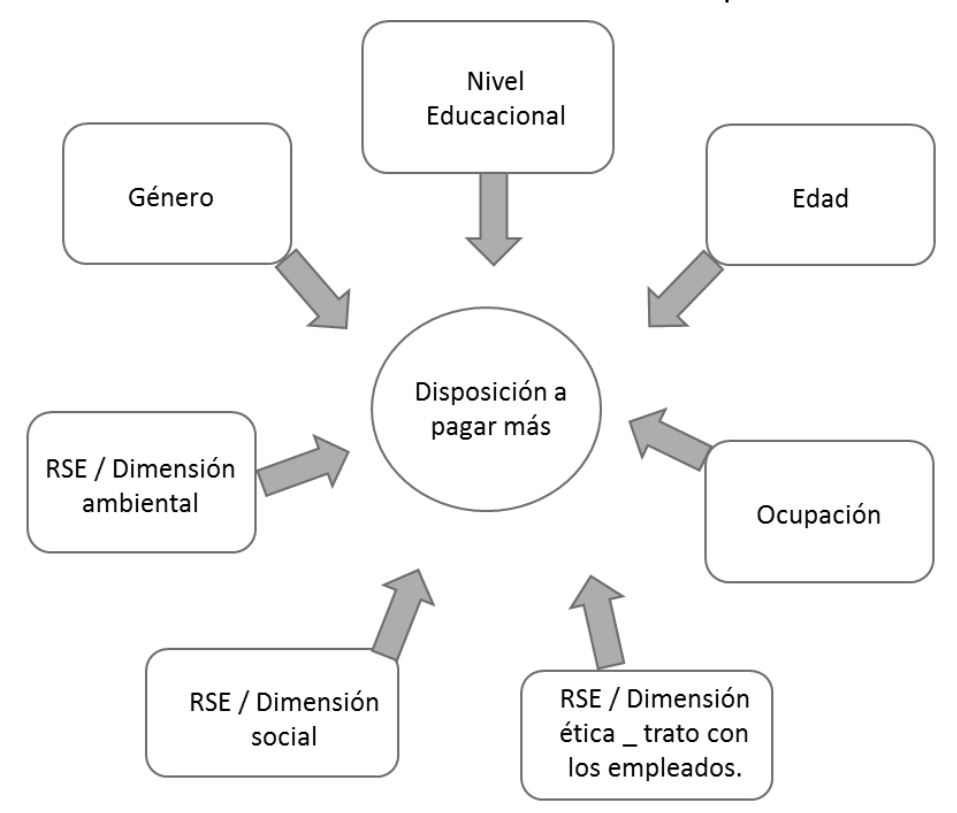

Fuente: elaboración propia

Teniendo en cuenta la Socially Resposible Purchase and Disposal (SRPD) (Webb et al., 2008), se tomó como referencia las dimensiones de la (RSE); en línea con Carroll (1979, 1999); Alvarado \& Schlesinger (2008) y Cancino \& Morales (2008), quienes contemplan la existencia de cuatro dimensiones de la (RSE): la económica, la legal, la ética y la discrecional o filantrópica. A su vez, este estudio evaluó 3 dimensiones en concordancia con la (RSE).

El compromiso social, el ambiental y las prácticas laborales; correspondientes también a tres de las siete dimensiones que evalúa la ISO 26000 (participación y desarrollo de la comunidad, derechos humanos, prácticas laborales, medio ambiente, prácticas de justa operación, asunto de consumidores y gobernanza).

Lo anterior, teniendo en cuenta que, en la revisión teórica, estas variables mostraron ser las de mejor desempeño e impacto en la disposición de los consumidores a pagar más por un producto 0 marcas, que manejen programas de responsabilidad empresarial.

ว
Variable dependiente:

CR: "Estoy dispuesto a pagar un poco más por aquellas marcas/productos que son respetuosas social y ambientalmente",

Variables independientes:

Dimensión ambiental 
CR1: "Procuro comprar productos que son amigables con el medioambiente".

CR2: "Procuro comprar a empresas/marcas que se comportan respetuosamente con el medioambiente".

Dimensión ética

CR3: "Cuando salgo de compras, intento comprar en aquellas empresas que respetan los derechos laborales de sus empleados".

\section{Dimensión social}

CR4: "Hago un esfuerzo por comprar a aquellas empresas que pagan un salario digno a todos sus empleados".

CR5: "Si me entero de que una empresa/almacén abusa laboralmente de sus empleados (horarios excesivos, sueldos insuficientes, etc.), evito comprar en ella".

Variables Demográficas:

Género, actividad, rango de edad y género y nivel de educación.

El alcance de la presente investigación es causal con un enfoque cuantitativo, basado en la aplicación de encuestas a nivel nacional para la recogida de la información, el trabajo de campo fue realizado por la empresa B\&Optimos durante el segundo semestre de 2015, con un muestreo no probabilístico en centros de gran afluencia.

Las variables de (RSE) objeto de estudio en la presente investigación, se midieron a través de escalas (SRPD), de la cual a juicio de expertos se seleccionaron las frases actitudinales de mayor relación con el tema, adaptándolas al contexto y cultura colombiana, mediante los correspondientes pretest cualitativos y cuantitativos; se tomaron dos frases actitudinales de la escala para medir la dimensión ambiental, una para la dimensión ética_ prácticas laborales y dos para la dimensión ambiental, frases actitudinales que fueron tratadas cada una como variables independientes, para un total de 5 variables.

Dichas variables fueron incluidas en un cuestionario que se aplicó a una muestra de 400 participantes de las principales ciudades del país (Bogotá, Medellín, Cali y Barranquilla), mayores de 18 años del nivel socioeconómico del 3 al 6 , implementando un diseño muestral estratificado por género y nivel socioeconómico.

El estudio se realizó con una confiabilidad del 95\% y $5 \%$ de margen de error, teniendo en cuenta el Universo total de la población colombiana según el DANE fue de 48.010.049 personas, distribuidas proporcionalmente para cada ciudad; 100 en Cali, 100 en Bogotá, 100 en Barranquilla y 100 en Medellín.

Esta muestra garantizó la representatividad de la muestra en los términos aceptados y validados de acuerdo con la literatura (Román \& Cuestas, 2008; Román, 2010). Para la medición de los constructos, se empleó una escala multi-ítem tipo Likert siguiendo un formato de 5 puntos Likert determinados.

\section{RESULTADOS:}

Es evidente una mayor incidencia de la dimensión; social, ética y ambiental en la disposición de los consumidores colombianos a pagar más, respecto a las variables demográficas.

\section{Contratación de Hipótesis:}

H1: Existe influencia entre los programas de responsabilidad social empresarial y la disposición de los consumidores a pagar por marcas / productos que realicen prácticas de responsabilidad social empresarial. 
Ajuste del Modelo de Regresión Logística:

Se ajustó un modelo de regresión logística, este tipo de modelo se encuadra dentro de los denominados Modelos Lineales Generalizados (Hosmer \& Lemeshow, 2000), donde la variable dependiente (VD) se transformó en una variable dicotómica, donde los puntajes 4 y 5 señalan los éxitos (1), mientras que cualquiera de los demás posibles valores señalan fracaso $(0)$. El resultado del ajuste se presenta a continuación.

\section{Fuente: elaboración propia}

Del cuadro anterior se puede deducir, según la probabilidad asociada a las pruebas del Chi2, que las variables que más aportan en la explicación de la VD, son CR1 y CR2, asociadas a la dimensión ambiental de la (RSE).

Las siguientes tablas proporcionan varios indicadores de la calidad del modelo (o calidad del ajuste). Se desea evaluar si las variables independientes CR1, CR2, CR3, CR4, CR5 y Variables Demográficas, proporcionan una cantidad de información significativa para explicar la variable binaria CR o dependiente.

En este caso, como la probabilidad es inferior a 0.0001 , se puede deducir que las variables proporcionan una cantidad significativa de información.

Tabla 1: Modelo de Regresión Logística

\begin{tabular}{|c|c|c|c|}
\hline Fuente & B & Chi-cuadrado de Wald & $\mathrm{Pr}>\mathbf{C h i}^{2}$ \\
\hline Intersección & $-72,114$ & 42,2784 & $<0,0001$ \\
\hline CR1 & 0,2682 & 13,1281 & 0,0003 \\
\hline CR2 & 0,2709 & 12,7541 & 0,0004 \\
\hline CR3 & $-0,1423$ & 1,1970 & 0,2739 \\
\hline CR4 & $-0,2596$ & 0,1168 & 0,7325 \\
\hline CR5 & $-0,0300$ & 2,9456 & 0,0861 \\
\hline GENERO-1 & & & \\
\hline GENERO-2 & $-0,4847$ & 0,0020 & 0,9643 \\
\hline ACTIVIDAD-2 & & & \\
\hline ACTIVIDAD-1 & $-11,403$ & 0,5177 & 0,4718 \\
\hline ACTIVIDAD-4 & $-0,5204$ & 0,4516 & 0,5016 \\
\hline ACTIVIDAD-3 & $-0,4641$ & 0,3391 & 0,5603 \\
\hline EDAD-2 & & & \\
\hline EDAD-1 & $-0,4554$ & 0,6450 & 0,4219 \\
\hline EDAD-3 & $-0,3383$ & 0,7933 & 0,3731 \\
\hline ESTUDIO-2 & & & \\
\hline ESTUDIO-3 & $-10,712$ & 2,4233 & 0,1195 \\
\hline ESTUDIO-1 & $-12,384$ & 3,2611 & 0,0709 \\
\hline
\end{tabular}




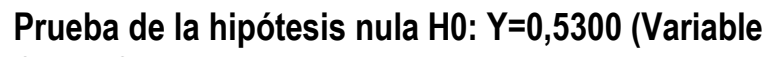
CR13_CB)

Tabla 2: Ajuste de la prueba $\mathrm{Chi}^{2}$

\begin{tabular}{l|c|c|c|}
\hline Estadística & GDL & Chi-cuadrado & Pr $>$ Chi \\
\hline $\mathbf{- 2}$ & 14 & 191,5446929 & $<0,0001$ \\
$\begin{array}{l}\text { Log(Verosimilitu } \\
\text { d) }\end{array}$ & & & \\
\hline Score & 14 & 113,0208135 & $<0,0001$ \\
\hline Wald & 14 & 81,53567168 & $<0,0001$ \\
\hline
\end{tabular}

Fuente: elaboración propia

Por último; se revisa el aporte a la VD de las independientes, sin las variables demográficas

Tabla 3: Ajuste modelo de regresión logística

\begin{tabular}{|c|c|c|c|}
\hline Fuente & B & $\begin{array}{c}\text { Chi- } \\
\text { cuadrado } \\
\text { de Wald }\end{array}$ & $\begin{array}{c}\text { Pr } \\
\text { Chi }^{2}\end{array}$ \\
\hline Intercepción & $-7,8036$ & 76,5197 & $<0,0001$ \\
\hline
\end{tabular}

Fuente: elaboración propia

De la tabla anterior se puede deducir, según la Probabilidad asociada a las Pruebas del Chi2, que las variables Independientes en su conjunto, sin las variables demográficas ayudan a potenciar el modelo. La constante del modelo tiene también un papel significativo en el ajuste de este modelo.

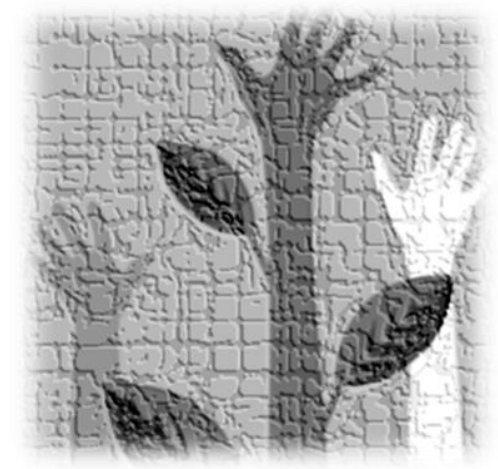

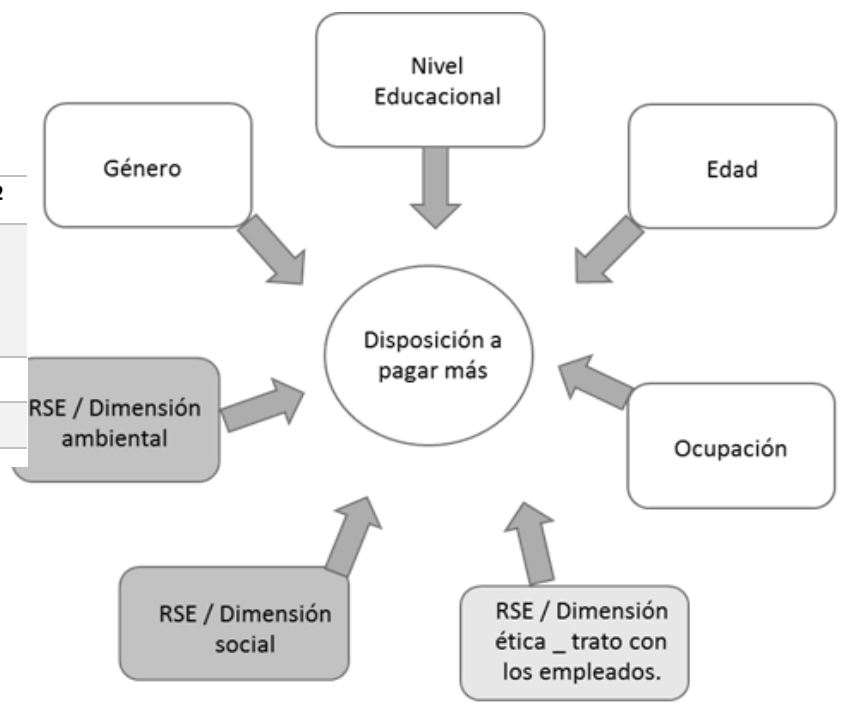

Fuente elaboración propia

Tabla 4: Prueba de la hipótesis nula HO: Y=0,5300 (VD_Vl's)

\begin{tabular}{|l|l|l|l|}
\hline Estadística & GDL & $\begin{array}{l}\text { Chi- } \\
\text { cuadrado }\end{array}$ & $\begin{array}{l}\text { Pr } \\
\text { Chi }^{2}\end{array}$ \\
\hline $\begin{array}{l}-2 \\
\text { Log(Verosimilitud) }\end{array}$ & 6 & 141,4458 & $\begin{array}{l}<, 0001 \\
0,001\end{array}$ \\
\hline Score & 6 & 117,5440 & $\begin{array}{l}< \\
0,0001\end{array}$ \\
\hline Wald & 6 & 84,9453 & $\begin{array}{l}< \\
0,0001\end{array}$ \\
\hline
\end{tabular}

Fuente: Elaboración propia

El valor más importante es el estadístico Chi^2 asociado al Log Ratio (L.R.), esto es el equivalente de la prueba $\mathrm{F}$ de Fisher del modelo lineal; en este caso se desea evaluar si las variables independientes proporcionan una cantidad de información significativa para explicar la variable binaria VD.

En este caso, como la probabilidad es inferior a 0.0001 , se puede deducir que las variables proporcionan una cantidad significativa de información.

En la siguiente tabla se presenta la prueba de bondad de ajuste de Hosmer-Lemeshow junto con su prueba Chi^2. 
Bajo un alpha del $5 \%$, se puede apreciar que el ajuste no es estadísticamente significativo, implicando esto que el modelo no presenta una consistencia estable; por lo tanto, no es conveniente la construcción del modelo.

\section{Tabla 5: Prueba de Hosmer-Lemeshow (Variable CR13_CB)}

\begin{tabular}{|c|c|c|c|}
\hline Estadística & $\begin{array}{c}\text { Chi- } \\
\text { cuadrado }\end{array}$ & GDL & $\begin{array}{c}\text { Pr }> \\
\text { Chi }^{2}\end{array}$ \\
\hline $\begin{array}{c}\text { Estadística de } \\
\text { Hosmer- } \\
\text { Lemeshow }\end{array}$ & 7,1288 & 8 & 0,5228 \\
\hline
\end{tabular}

Fuente: Elaboración propia

\section{CONCLUSIONES:}

Teniendo en cuenta las variables de segmentación más generales de las cuales tradicionalmente habla la literatura como son; las variables demográficas, sociodemográficas, geodemográficas; este estudio no pretende desvirtuar su importancia y mucho menos en el tema tratado en esta investigación, pero teniendo claro que la segmentación es un híbrido, en donde confluyen diferentes variables sin llegar a caer en la exageración de las variables consideradas para la segmentación, teniendo en cuenta la representatividad y la suficiencia de los targets para que sean atractivos en la elaboración de una estrategia de mercadeo; es importante considerar que no todas las veces en el proceso de segmentación, se inicia o priman este tipo de variables, precisamente éste es el principal hallazgo de la investigación y lo que hace más atractivo el considerar los programas de responsabilidad social empresarial dentro de los planes de mercadeo; puesto que es una variable transversal a cualquier rango de edad, género 0 la ocupación entre otras variables demográficas; obviamente considerando las diferentes formas que se deben manejar, de acuerdo a cada uno de los grupos teniendo en cuenta que presentan características diferenciadoras.

Por otra parte; al no encontrarse influencia directa entre las variables demográficas, y la disposición de los consumidores a pagar más por productos socialmente responsables, pero sí que manejen los programas de responsabilidad social empresarial dentro de sus estrategias de marcas; ya sea por convicción o por cumplimiento legal, se puede concluir que ésta variable se convierte en una exigencia del consumidor actual, motivados por un nuevo estilo de vida, que se sustenta muy bien con la literatura revisada y expuesta, no es un tema 0 actitud solamente de los Colombianos; es un tema de consciencia a nivel universal, que con el paso del tiempo dejará de ser una norma de cumplimiento legal para algunas empresas y será parte de la cotidianidad; por lo tanto, el resultado de esta investigación es una voz de alerta para aquellas marcas que aún no consideran esta estrategia dentro de sus planes de mercadeo como parte de su ADN; puesto que el consumidor, genera mayores niveles de cercanía con aquellas marcas en las cuales perciben un trabajo real que le aporta a su calidad de vida, más allá de un producto que satisface muy bien una necesidad "per se", sin pensar en el impacto de todos los Stakeholder.

\section{REFERENCIAS:}

Alvarado, Herrera; Alejandro, Schlesinger; Díaz, María Walesska. (2008). Dimensionalidad de la Responsabilidad Social Empresarial percibida y sus efectos sobre la imagen y la reputación: Una aproximación desde el modelo de Carroll. Estudios Gerenciales, [en linea] 24 (Julio-Septiembre) : [Fecha de consulta: 20 de junio de 2019]

Disponible en:<http://www.redalyc.org/articulo.o $a ? i d=21210802>$ ISSN 0123-5923 
Alzate, Jaime \& Orozco, Jaime Alberto. (2015), Diseño y Responsabilidad Social Empresarial en la planeación estratégica de la marca. Revista Kepes, 12, pp. 133- 155.

Arredondo, Florina Guadalupe; Maldonado, Verónica del Carmen \& De La Garza, Jorge. (2010). Consumers and their buying decision making based on price and information about corporate social responsibility (CSR). Case study: Undergraduate students from a private university in Mexico. Estudios Gerenciales, 26 (117), pp. 103-117. Disponible en: http://www.scielo.org.co/pdf/eg/v26n117/v26 n117a06.pdf

Amezcua, Bernardo, Briseño, Arturo, Ríos, Teresa, \& Ayala, Edgardo. (2018). La disposición a pagar más por productos vinculados a la RSE: evidencia de un análisis conjunto en México. Contaduría y administración, 63(2) HYPERLINK Disponible en:https://dx.doi.org/10.22201/fca.24488410e .2018 .1013

Cai, Zhen and Francisco X. Aguilar (2013), "Metaanalysis of Consumer's Willingness-toPay Premiumsfor Certified Wood Products," Journal of Forest Economics, 19 (January), pp. 15-31.

Cancino, Christian \& Morales, Mario. (2008), Responsabilidad social empresarial. Serie Documento Docente. Universidad de Chile. Santiago de Chile, Chile. Disponible en:

http://repositorio.uchile.cl/bitstream/ handle/2250/122747/Cancino_Morales_2008 .p df?sequence $=1$

Carroll, Archie. (1979), A Three-Dimensional Conceptual Model of Social Performance. Academy of Management Review. 4. 497-505. 10.2307/257850.

Carroll, Archie. (2015), Corporate social responsibility: The centerpiece of competing and complementary frameworks. Organizational Dynamics, 44, pp. 87-96

Castelló, A. (2010). Estrategias empresariales en la Web 2.0. Las redes sociales online.

Alicante: Editorial ECU.

Havas Worldwide (2016). Superbrands 2016: los consumidores y el futuro de los modelos empresariales. Disponible en: http://www.compromisoempresarial. com/rsc/2016/05/el-58-de-los-consumidoresse-interesa-por-el-impacto-social-yambiental-de-las-marcas/

Licandro, Oscar; Alvarado-Peña, Lisandro; SansoresGuerrero, Edgar \& Navarrete, Juana. (2019), Responsabilidad Social Empresaria: Hacia la conformación de una tipología de definiciones. Revista Venezolana de Gerencia, 24. pp. 281-299. Recuperado en: http://produccioncientificaluz.org/ind ex.php/rvg/article/view/23843 Fecha de consulta:20/06/2019

López \& Peñalosa (2018). El consumo socialmente responsable como estilo de vida de los colombianos y su aplicación al marketing. Revista Espacios.Vol. 39 ( $\mathrm{N}^{\circ} 34$ ). Disponible

en: https://www.revistaespacios.com/a18v39n34/ a18v39n34p03.pdf

Marquina Feldman, \& Percy-Reficco, Ezequiel. (2015), Impacto de la responsabilidad social empresarial en el comportamiento de compra y disposición a pagar de consumidores bogotanos. Estudios Gerenciales [en línea] 31 (octubre-diciembre): Disponible en:<http://www.redalyc.org/articulo.oa?id=21 243557003> ISSN 0123- 5923 Fecha de consulta: 20 de junio de 2019 
Osorio, L. M. B., Andrade, J. M. M., \& Mina, L. G. (2015). EXPERIENCIAS DEL BRANDING COMO ESTRATEGIA PARA EL POSICIONAMIENTO EN EL MERCADO INTERNACIONAL. FACE: Revista de la Facultad de Ciencias Económicas y Empresariales, 15(2), 91-103. http://revistas.unipamplona.edu.co/ojs vicein ves/index.php/FACE/article/viewFile/1812/80 $\underline{2}$

Otalvaro, M. J. N. (2016). Diagnóstico del clima laboral y organizacional de los conductores de los campactadores de la empresa de aseo de Medellín-emvarias. FACE: Revista de la Facultad de Ciencias Económicas y Empresariales, 15(2), 57-69. http://revistas.unipamplona.edu.co/ojs_vicein ves/index.php/FACE/article/download/1861/8 $\underline{26}$

Orozco Toro, J. A., \& Alzate Sanz, J. E. (2018). Stakeholders: Strategic Actors in Brand Building. Anagramas rumbos y sentidos de la comunicación, 16(32), 95-109. https://doi.org/10.22395/angr.v16n3 $2 a 6$

Pérez, A. \& Rodriguez, I. (2015). How customers construct corporate social responsibility images: Testing the moderating role of demographic characteristics BRQ. Business Research Quarterly 18 (1), pp. 127-141.

Solari, Estefanía. \& Pacheco Ortiz, Diana. (2015), Disposición a pagar por atributos de responsabilidad social empresarial. Un estudio aplicado a jóvenes de Medellín. Revista Virtual Universidad Católica del Norte, 46, pp. 187- 199. Disponible en: http://revistavirtual.ucn.edu.co/index. php/RevistaUCN/article/view/708/1235. ISSN 0124-5821

Tian, Zhilong; Wang, Rui \& Yang, Wen. (2011), Consumer responses to Corporate Social Responsibility. (CSR) in China. Journal of Business Ethics, 101, pp. 197-212.
Disponible en: https://uu.divaportal.org/smash/get/diva2:1134668/ FULLTEXT01.pdf

Vicente, M.A. y Mediano, L., 2002, "Propuestas para una segmentación estratégica del mercado ecológico". Cuadernos de Gestión, Vol. 2, No. 1, pp. 1130.

Webb, Deborah \& Mohr, Lois \& E. Harris, Katherine. (2008). A re-examination of socially responsible consumption and its measurement. Journal of Business Research. $61 . \quad \mathrm{pp}$. 91-98. 10.1016/j.jbusres.2007.05.007.

World Econmic Forum. (2017), The Global Shapers Survey. \#shaperssurvey. Dispoible en: http://www.shaperssurvey2017.org/s tatic/data/WEF_GSC_Annual_Survey_2017. $\mathrm{p} \quad \mathrm{df}$

Zhilong, T., Rui, W., \& Wen, Y. (2011). Consumer Responses to Corporate Social Responsibility (CSR) in China. Journal of Business Ethics (2011) 101:197212 _ Springer 2011 DOI 10.1007/s 10551010-0716-6 